\title{
Curcumin as a potential therapeutic option for NAFLD and other metabolic diseases: need for establishing the underlying mechanism(s) of action
}

\author{
Shobha Ghosh ${ }^{1}$ (D)
}

Received: 15 January 2019 / Accepted: 20 February 2019 / Published online: 19 March 2019

(c) Asian Pacific Association for the Study of the Liver 2019

The natural polyphenol, curcumin, the most widely studied component of the Asian spice Turmeric, is shown to have potent anti-oxidant, anti-inflammatory, anti-bacterial and anti-neoplastic activities [1-4]. These varied beneficial effects have been largely examined using cell culturebased assays in vitro where curcumin is in direct contact with the target cells. However, limited oral bioavailability of curcumin and levels of curcumin or its metabolites in systemic circulation below the detection limits of most analytical methods hinder the establishment of a cause-effect relationship between orally administered curcumin and the observed beneficial effects in vivo [5]. Furthermore, results from several small-scale clinical studies/trials have not been entirely consistent complicating an already difficult problem. Nonetheless, ever increasing published data and widespread recognition of curcumin as a dietary supplement warrant a systematic review of existing literature, preferably in the context of a specific disease process.

In this issue of the Journal, Wei et al. [6] report a metaanalysis of randomized controlled trials (RCTs) examining the effects of curcumin on the metabolic parameters of nonalcoholic fatty liver disease (NAFLD). Out of 99 studies identified by literature searches, only four studies met the stringent inclusion criteria namely, RCT or parallel trial, included subjects $>18$ years of age, assessment of NAFLD (grades 1-3) by liver ultrasound and the fact that the intervention group received curcumin and control group received a placebo. Curcumin supplementation significantly lowered plasma LDL-C, triglycerides, fasting blood sugar, HOMAIR, body weight and AST levels. However, the observed lowering of total plasma cholesterol, HDL-C, HbA1c, ALT or insulin levels by curcumin did not reach statistical

Shobha Ghosh

shobha.ghosh@vcuhealth.org

1 Virginia Commonwealth University Medical Center, Richmond, VA, USA significance. Detailed analyses on the effects of curcumin on the severity of hepatic steatosis, an important parameter for NAFLD, could not be performed due to the limited number of available studies. Despite another limitation noted by the authors, namely small number of patients included in most studies, these are promising/encouraging data especially considering the poor bioavailability of orally administered curcumin and raise two intriguing possibilities:

1. Will enhancing the bioavailability of curcumin increase the magnitude of its beneficial effects? In recent years, multiple formulations of curcumin have been developed to increase its absorption and bioavailability. However, no clinical trials or studies have been conducted examining the effects of curcumin or these modified versions in parallel with unmodified curcumin precluding the direct comparative efficacy. For example, addition of piperine is thought to increase curcumin bioavailability [7], but while RCT examining the effects of this combination was placebo controlled, it did not include a curcumin alone group to facilitate the evaluation of the effects of piperine addition and resulting curcumin bioavailability on changes in the metabolic parameters examined [8]. Furthermore, circulating levels of curcumin or curcumin metabolites are seldom quantified in any clinical study or trial precluding the direct examination of any changes in bioavailability and observed metabolic effects. More appropriately designed studies are required to establish a causative link between changes in bioavailability of curcumin and its observed beneficial effects. In this regard, it would be critical to focus on one disease at a time and collect data on all parameters relevant to the likely cause(s) of the disease and correlate them with plasma levels of curcumin and/or its metabolites to firmly establish the much needed cause-effect relationship to move the use of curcumin to therapeutic arena. 
Fig. 1 Schematic to show the likely mechanisms underlying the observed beneficial effects of curcumin and how these need to be established by future studies

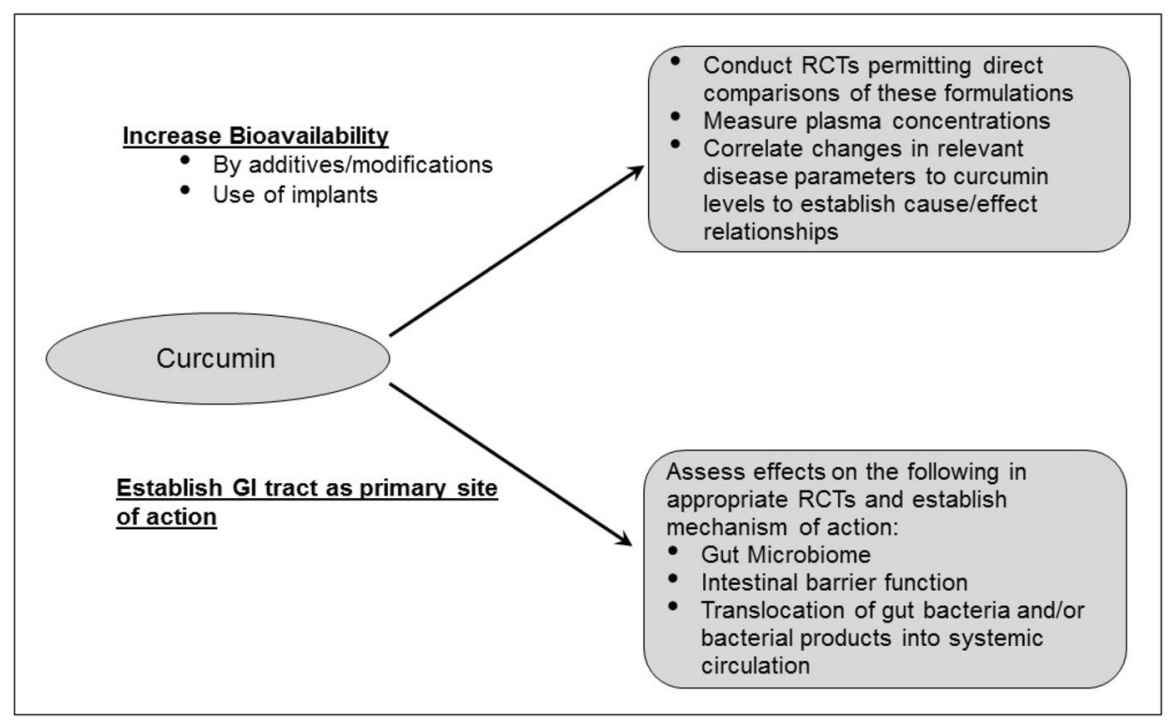

2. Are the beneficial systemic/metabolic effects of orally administered curcumin not directly related to the amount of Curcumin absorbed but rather due to action of curcumin in the gastrointestinal (GI) tract? It is noteworthy that clinical use of curcumin is most advanced in the treatment of inflammatory bowel disease, ulcerative colitis and other GI tract-related diseases [9]. In addition, data from several pre-clinical studies provide strong evidence for the direct effects of curcumin on the GI tract. Zou et al. [10] reported that curcumin acts as a potent anti-atherogenic agent by inhibiting cholesterol absorption in mice. Feng et al. [11] demonstrated that curcumin dramatically shifted the overall structure of the high fat diet-disrupted gut microbiota toward that of lean rats fed a normal diet and attributed these changes as the likely mechanism for the observed attenuation of hepatic steatosis. Curcumin-mediated improvement of metaflammation-related diseases such as diabetes, atherosclerosis or chronic kidney disease is also likely due to the effects of curcumin in improving intestinal barrier function [12] and limiting the release of bacterial endotoxin [13] and other bacterial products into systemic circulation [14]. Similar attenuation of systemic inflammation by oral curcumin might also underlie the beneficial effects on other inflammatory diseases such as arthritis. While studies with animal models of metaflammationrelated diseases provide additional evidence for the GI tract-focused effects of curcumin, it is critical to monitor similar parameters (e.g., intestinal permeability, plasma levels of intestinal bacteria-derived products, gut microbiome analyses) in clinical studies/trials to advance our current understanding of the mechanism of action of curcumin.
Incomplete understanding of how curcumin exerts its effects in vivo is one of the major hurdles in developing curcumin as a therapeutic strategy, especially for diseases such as NAFLD where new treatment strategies are urgently needed. Meta-analyses of RCTs, such as the one by Wei et al. [6], are an important first step to establish the beneficial effects of oral supplementation on individual metabolic parameters needed to be modified in a given disease process. Verification of several other aspects related to curcumin bioavailability and/or primary site of action (Fig. 1) in humans is obviously most important. The technical issues with detecting low levels of curcumin in systemic circulation are being resolved and in addition to modified formulations, controlled systemic release via implants is also being explored [15] and is likely to provide correlation between circulating levels of curcumin and observed effects. Significant advances have also been made in determining changes in gut microbiome as well as assessment of intestinal barrier function in clinical samples that will facilitate the evaluation of action of curcumin via modulation of the GI tract. Together these approaches open new opportunities to close the gap between uses of curcumin as a nutritional supplement to its use as therapy either by itself or in combination with other established therapies.

\section{Compliance with ethical standards}

Conflict of interest Shobha Ghosh declares no conflict of interest. 


\section{References}

1. Aggarwal BB, Harikumar KB. Potential therapeutic effects of curcumin, the anti-inflammatory agent, against neurodegenerative, cardiovascular, pulmonary, metabolic, autoimmune and neoplastic diseases. Int J Biochem Cell Biol 2009;41:40-59

2. Schraufstatter E, Bernt $\mathrm{H}$. Antibacterial action of curcumin and related compounds. Nature 1949;164:456

3. Hatcher H, Planalp R, Cho J, Troti FM, Troti SV. Curcumin: from ancient medicine to current clinical trials. Cell Mol Life Sci 2008;65:1631-1652

4. Aggarwal BB. Pharmacological basis for the role of curcumin in chronic diseases: an age-old spice with modern targets. Trends Pharmacol Sci 2009;30:85-94

5. Nelson KM, Dahlin JL, Bisson J, Graham J, Pauli GF, Walters MA. The essential medicinal chemistry of curcumin - mini perspective. J Med Chem 2017;60:1620-1637

6. Wei Z, Liu N, Tantai X, Xing X, Xiao C, Chen L, Wang J (2018) The effects of curcumin on the metabolic parameters of non-alcoholic fatty liver disease: a meta-analysis of randomized controlled trials. Hepatol Int. https://doi.org/10.1007/s12072-018-9910-x

7. Shoba G, Joy D, Joseph T, Majeed M, Rajendran R, Srinivas PS Influence of piperine on the pharmacokinetics of curcumin in animals and human volunteers. Planta Med 1998;64:353-356

8. Panahi Y, Khalili N, Sahebi E, Namazi S, Simental-Mendía LE, Majeed M, et al. Effects of curcuminoids plus piperine on glycemic, hepatic and inflammatory biomarkers in patients with type 2 diabetes mellitus: a randomized double-blind placebo-controlled trial. Drug Res 2018;68:403-409 (Stuttg)

9. Iqbal U, Anwar H, Quadri AA. Use of curcumin in achieving clinical and endoscopic remission in ulcerative colitis: a systematic review and meta-analysis. Am J Med Sci 2018;356:350-356
10. Zou J, Zhang S, Li P, Zheng X, Feng D. Supplementation with curcumin inhibits intestinal cholesterol absorption and prevents atherosclerosis in high-fat diet-fed apolipoprotein E knockout mice. Nutr Res 2018;56:32-40

11. Feng W, Wang H, Zhang P, Gao C, Tao J, Ge Z, et al. Modulation of gut microbiota contributes to curcumin-mediated attenuation of hepatic steatosis in rats. Biochim Biophys Acta Gen Subj 2017;1861:1801-1812

12. Ghosh SS, He H, Wang J, Gehr TW, Ghosh S. Curcumin-mediated regulation of intestinal barrier function: the mechanism underlying its beneficial effects. Tissue Barriers 2018;6(1):e1425085

13. Ghosh SS, Gehr TW, Ghosh S. Curcumin and chronic kidney disease (CKD): major mode of action through stimulating endogenous intestinal alkaline phosphatase. Molecules 2014;19:20139-20156

14. Ghosh SS, Righi S, Krieg R, Kang L, Carl D, Wang J, et al. High fat high cholesterol diet (western diet) aggravates atherosclerosis, hyperglycemia and renal failure in nephrectomized ldl receptor knockout mice: role of intestine derived Lipopolysaccharide. PLoS One 2015;10:e141109

15. Bansal SS, Kausar H, Vadhanam MV, Ravoori S, Gupta RC. Controlled systemic delivery by polymeric implants enhances tissue and plasma curcumin levels compared with oral administration. Eur J Pharm Biopharm 2012;80:571-577

Publisher's Note Springer Nature remains neutral with regard to jurisdictional claims in published maps and institutional affiliations. 